\title{
Failure Modes, Mechanisms, and Effects Analysis for LED Backlight Systems used in LCD TVs
}

\author{
Jiajie Fan K.C.Yung \\ Department of Industrial and Systems Engineering, \\ The Hong Kong Polytechnic University, \\ Hong Kong, China \\ e-mail: jay.fan@polyu.edu.hk
}

\author{
Michael Pecht \\ Prognostics and Health Management Centre \\ City University of Hong Kong \\ Hong Kong, China \\ e-mail: mgpecht@cityu.edu.hk
}

\begin{abstract}
Compared to conventional light sources, LED backlight systems, consisted by LED backlight units (BLUs) and drivers, provide a wide range of performance benefits for Liquid crystal display (LCD) TVs. And the failure of this electronic system directly determines the life of LCD TVs. So the growing interests have been focused on the prognostic and health management (PHM) for this electronic system. As the first step of physics-of-failure (PoF) based PHM approach widely used in reliability assessment, failure modes, mechanisms, and effects analysis (FMMEA) was used in this paper to identify the potential failure mechanisms and associated failure sides in LED backlight systems.
\end{abstract}

Keywords- FMMEA; LED Backlight; LCD TVs; Electronic Assembly

\section{INTRODUCTION}

Recently, Liquid crystal displays (LCDs) have been widely used as information displays, including LCD-TVs, laptop monitors, mobile phones and car navigation systems, and also have become an essential part of our lives[1]. As they can't self-laminate, LCDs employ a backlight unit (BLU) as their light source which consumes about $90 \%$ of the total power and $40 \%$ of the material costs. So as to broaden LCDs' market shares in displays, higher brightness, lower power consumption, wider color gamut and lengthening the lifetime have been considered as critical requirements for the BLU used in LCDs.

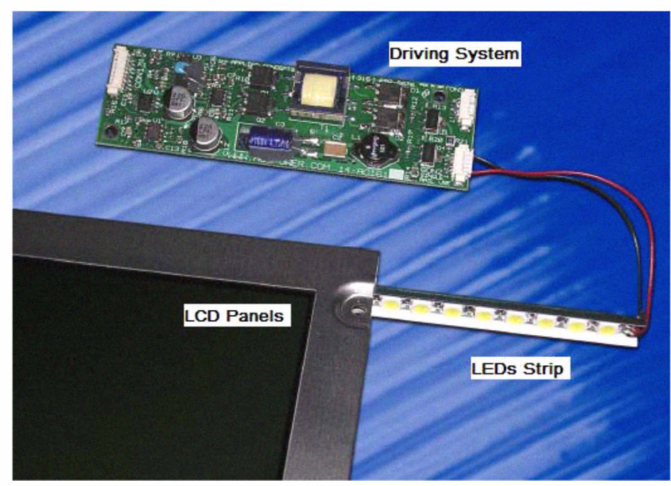

Fig 1 LED Backlight Systems

Compared to the traditional backlight source-Cold Cathode Fluorescent Lamps (CCFLs), Light Emitting Diodes (LEDs) possess many advantages, i.e longer lifetime $(>50.000$ hrs), wider operation temperature range, lower working voltages and mercury and lead free, and have rapidly entered into the LCD display market[2]. In addition, to improve the display quality, an advanced and reliable backlight driving system (normally is an electronic assembly) is integrated into the whole module consisting of an electronic system (Fig. 1). And the reliability of this backlight electronic system determines the LCD TVs' life. Therefore, how to accurately predict the Remaining Useful Lifetime (RUL) of the LED BLU electronic system in the design period is becoming one of the key projects to popularize this novel product.

In order to do reliability assessments with physics-offailure (PoF) based on prognostics and health management (PHM) approach for this electronic system, LED BLUs materials and driving electronic components used in this system were first analyzed in this paper and then failure modes, mechanisms, and effects analysis (FMMEA) were used to identify the potential failures.

\section{LED BLU MATERIALS AND DRIVING ELECTRONIC COMPONENTS ANALYSIS}

In today's LCD display market, there are two types of LED backlights: (1) direct backlight in which LEDs arranged in a dense array behind the LCD panels (Fig 2 a); (2) indirect backlight or edge light which is constructed with four LED strips on the edges and can reduce the thickness to about $10 \mathrm{~mm}$ and generate uniform backlight by means of a light guiding plate(Fig $2 \mathrm{~b}$ ). And three solutions are widely used to create white light: (1) blue LEDs with yellow phosphor (YAG: $\mathrm{Ce}^{3+}$ ) coatings; (2) UV LEDs combined with RGB phosphors; (3) RGB LEDs clusters (individual-colored-array type or multiple-chip-in-a-package type).

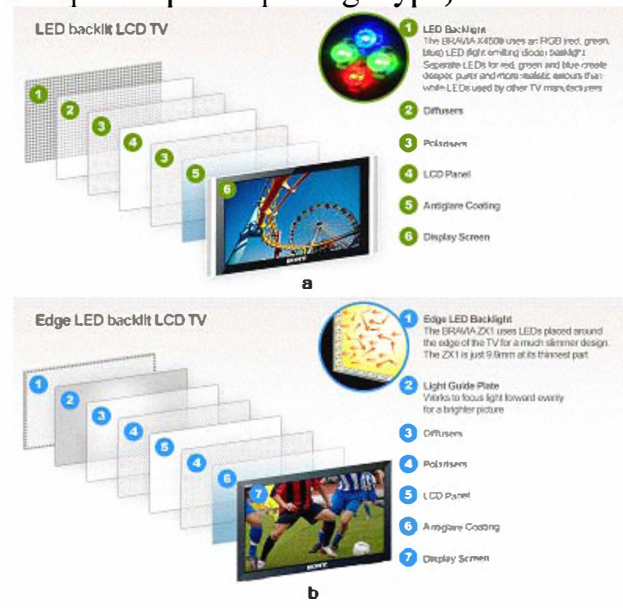

Fig 2 Two Types of LED Backlights used in LCD TVs (from Sony. CO., LTD|3|) 
Many electronics companies have proposed their LED BLUs for LCD backlighting. For example, LUMILED, the Netherlands, has put out High Power Luxeon ${ }^{\mathrm{TM}}$ side-emitter devices which have a planar radiation pattern to improve color mixing. And the RGB LED BLUs were surface mounted to a metal core print circuit board (MCPCB) strip by the sequence of green, red-orange, blue, green..... [4]. OSRAM also introduced his six-lead MULTILED LRTB G6SG multi-chip SMD LED which contained three color chips $(625 \mathrm{~nm}$ red, $528 \mathrm{~nm}$ true-green and $458 \mathrm{~nm}$ blue) and was used to construct an edge light strip [5]. Table 1 shows the differences of two types LED BLUs packaged as a LCD backlight strip (Fig 1).

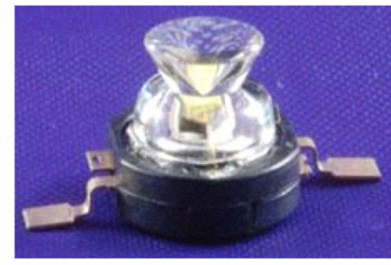

(a)

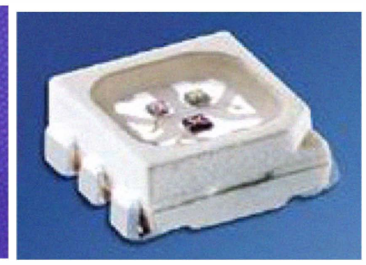

(b)
Fig 3 LED BLUs for LCD backlighting (a: Luxeon $^{\text {TM }}$, b: MULTILED LRTB G6SG)

Table 1 Technology differences between two types of LED

\begin{tabular}{|c|c|c|}
\multicolumn{3}{|c}{ BLUs } \\
\hline Package type & $\begin{array}{c}\text { Luxeon }{ }^{\text {TM }} \\
\text { leads }\end{array}$ & MULTILED LRTB G6SG \\
\hline $\begin{array}{c}\text { Assembly } \\
\text { Technology }\end{array}$ & Wire bonding & Six J-type leads \\
\hline $\begin{array}{c}\text { Optical } \\
\text { Efficiency(Lm/W) }\end{array}$ & $\begin{array}{c}\text { Red-orange: } 60 \\
\text { Green: } 46 \\
\text { Blue: } 5.5 \\
\text { White: } 37\end{array}$ & Wire bonding \\
\hline Chips & $\begin{array}{c}\text { All color chips } \\
\text { True green: } 36 \\
\text { Blue: } 11\end{array}$ \\
\hline Phosphor Coating & $\begin{array}{c}\text { Only available in } \\
\text { white type }\end{array}$ & $\begin{array}{c}\text { Thin film (Red), Thin } \\
\text { GaN(Blue, true green) }\end{array}$ \\
\hline PCB & MCPCB & No \\
\hline SMT Method & Soldering & MCPCB \\
\hline & & Soldering \\
\hline
\end{tabular}

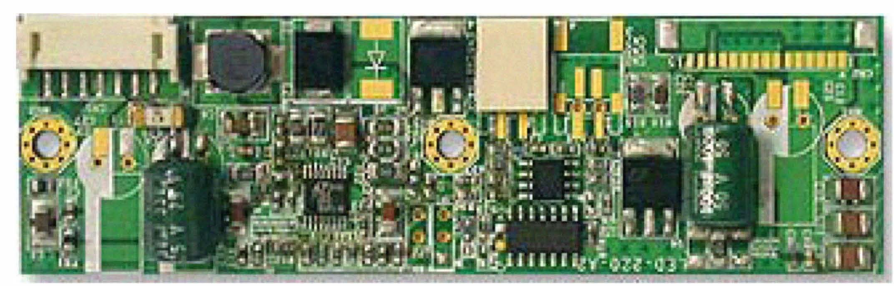

Fig 4 LED backlight driver (LED-252280)

Table 2 Types and functions of electronic components in LED driving systems

\begin{tabular}{|c|c|l|}
\hline & Types & \multicolumn{1}{c|}{ Functions } \\
\hline \multirow{4}{*}{$\begin{array}{c}\text { Active } \\
\text { Components }\end{array}$} & MOSFETs & $\begin{array}{l}\text { Electronic switches in switching and } \\
\text { linear LED driver circuits }\end{array}$ \\
\cline { 2 - 3 } & Bipolar Transistors & $\begin{array}{l}\text { Switching and linear LED driver } \\
\text { circuit }\end{array}$ \\
\cline { 2 - 3 } & Rectifier & Reverse polarity protection \\
\cline { 2 - 3 } Passive & $\begin{array}{c}\text { Voltage Clamping } \\
\text { Devices }\end{array}$ & Limit the voltage across a circuit \\
\cline { 2 - 3 } Components & Capacitors & Energy storage \\
\cline { 2 - 3 } & Inductors & $\begin{array}{l}\text { Energy storage in switching LED } \\
\text { driver circuits }\end{array}$ \\
\cline { 2 - 3 } & Resistors & $\begin{array}{l}\text { Provide impedance for fast transients } \\
\text { and surges }\end{array}$ \\
\hline
\end{tabular}

\begin{tabular}{|c|c|l|}
\hline & PCB & Electric and mechanical supports \\
\cline { 2 - 3 } & $\begin{array}{c}\text { Operational } \\
\text { Amplifiers }\end{array}$ & Frequency response \\
\cline { 2 - 3 } & Comparators & $\begin{array}{l}\text { Detect the current level in a sense } \\
\text { resistor }\end{array}$ \\
\hline
\end{tabular}

To control so many LED BLUs efficiently, driving systems are applied to LED backlight systems. Normally, today's LED backlight systems require the driver solution with the following features: (1) direct control of current; (2) high efficiency; (2) PWM dimming; (3) over-voltage protection; (4) load disconnect; (5) small size; (6) ease of use[6][7]. Fig 1 describes an LED backlight driving system as an electronic assembly which contains several active and positive electronic components integrated on the surface of the print circuit board. Fig 4 is a LED backlight driving board (LED-252280) produced by Spectrah[21]. This module has several features to control LED BLUs arrays: (1) high dimming ration (3000:1); (2) low profile and compact design; (3) constant LED current (280mA); (4) PWM/or DC voltage dimming control; (5) short circuit and overload protection; (6) external power on/off control. Table 2 lists all types of electronic components and their functions to driving LED BLUs strips.

\section{FAILURE MODES, MECHANISMS, AND EFFECTS ANALYSIS}

In electronics systems, a failure mode is the recognizable electrical symptom by which a failure is observed, i.e. circuit open or short. And each mode could be caused by one or more different failure mechanisms which could be driven by physical, chemical, mechanical and so on[8][11][12]. Failure mechanisms could be categorized to overstress (catastrophic) failure and wear-out (gradual) failure mechanisms[9]. Overstress failure arises as a result of a single load (stress) condition, which exceeds the threshold of a strength property. Relatively, wear-out failure occurs as a result of cumulative damage related to loads (stresses) applied over an extended period of time[10].

As discussed above, LED backlight systems were formed by LED BLUs strips and associated driver. But the driving system mentioned can be seen as a sub-system to supply and optimize the power to the LED BLUs strip module. So the ultimate failure modes for LED backlight systems can be categorized to: (1) light output off; (2) lighting Color changes; (3) luminous flux degradation. And the failures of driving system would contribute to the above three failure modes occurred in LED BLUs strip module and also can be defined as accompanying failure mechanisms. (Fig 6)

\subsection{Failures in LED BLUs strip section \\ 3.1.1. Chip level degradation}

Aforementioned, there were many types of LED chips used in BLUs (red, green, blue et al). But the common degradation mechanism of the active layer of LEDs is due to increased non-radiative recombination which lowers the optical output power and power efficiency. The responsible factors which contribute to the non-radiative recombination were proposed to[13]: 


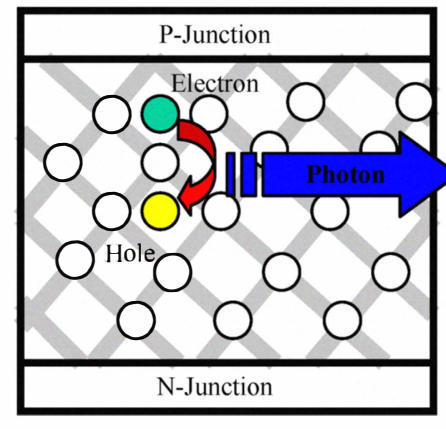

(a)

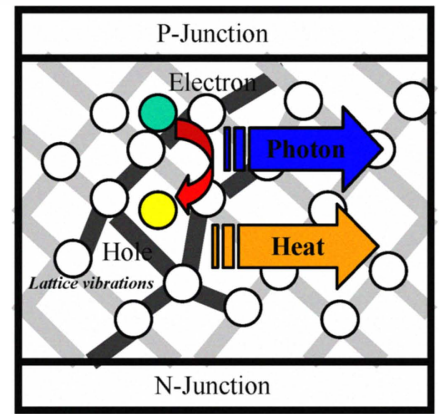

(b)

Fig 5 The mechanisms of radiative recombination (a) and non-radiative recombination (b)

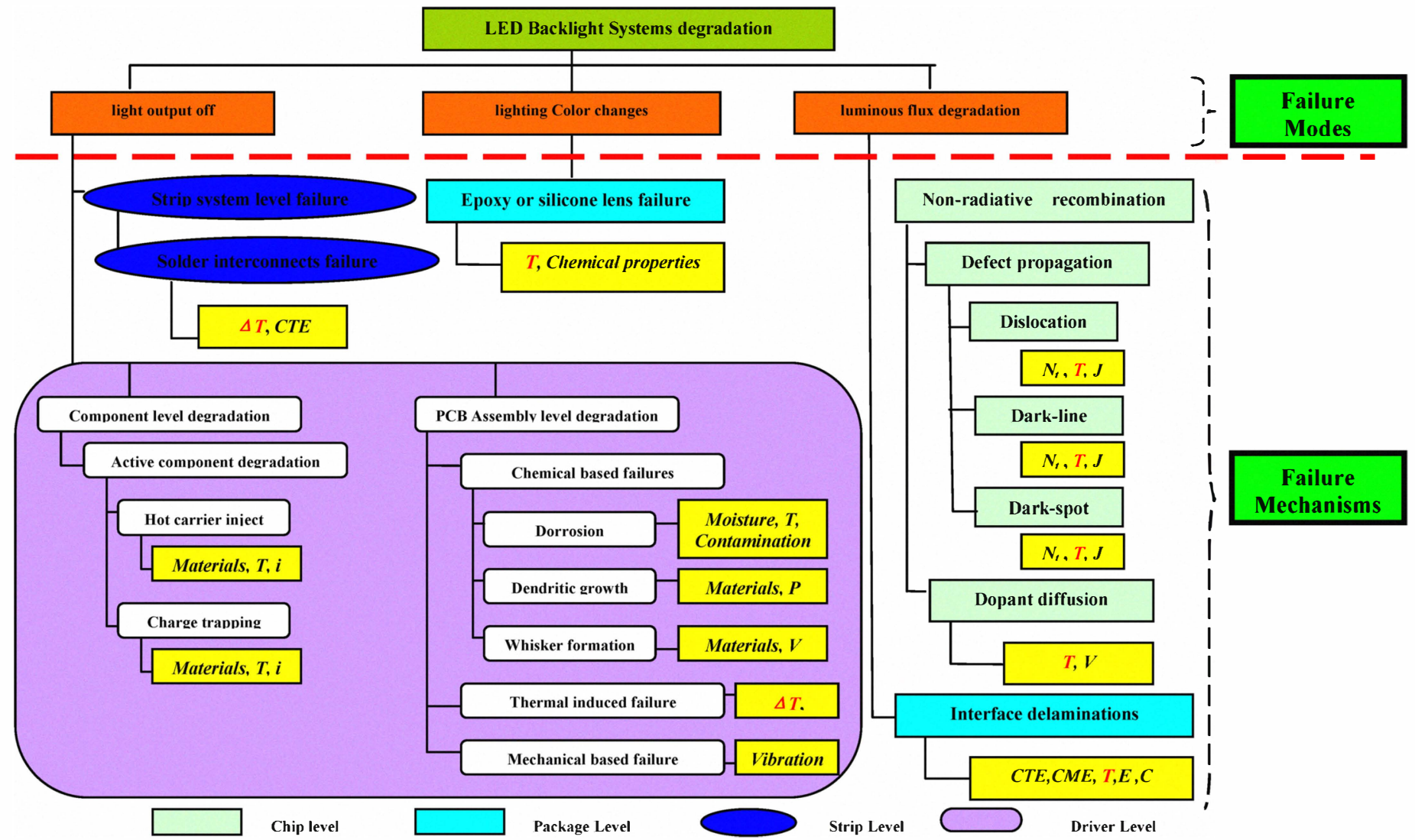

Fig 6 FMMEA for LED Backlight systems used in LCD TVs

( $\Delta T$ : Thernal Cycle; $N_{t}$ : the defect density; $J$ : Current Density; $V:$ Voltage; $E$ : Elastic Modulus; $C$ : Moisture Concentration; P: Pressure; i: Current)

(1) The defects (dislocation, dark-line and dark-spot) propagation is one of the suspects to increase the nonradiative recombination which converts most electron-hole recombination energy to heat by lattice atoms' vibrations[14][15][16]. (Fig 5)

A Carrier-Continuity equation (Equation 1) has been widely used to show the qualitative competition between radiative and non-radiative recombination occurred in the quantum well active region. As shown in equation 2 which expresses the non-radiative recombination coefficient by the Shockley-Hall-Read recombination rate, increasing defect density $N_{t}$ will contribute to the non-radiative recombination and relatively reduce the light output intensity for a certain value of the forward current[15].

$$
\begin{gathered}
\frac{d n}{d t}=\frac{J}{e d}-B n^{2}(t)-A n(t) \\
A=N_{t} \nu_{t h} \sigma
\end{gathered}
$$

where the $J / e d$ is the current injection rate, the $B n^{2}(t)$ accounts for the spontaneous emission rate ( or luminous radiative term), and the $A n(t)$ represents the non-radiative carrier captures at the defects. $A$ and $B$ are the non-radiative and radiative coefficient, respectively. $N_{t}$ is the defect density of traps, $v_{t h}$ is the carrier of thermal velocity and $\sigma$ the electron capture cross section

(2) Diffusion of dopants or impurities in the quantum well (QW) region also increases non-radiative recombination. In GaN-based LEDs, Mg dopant must be heavily doped on GaN epilayer to obtain a sufficient carrier density due to the high activation energy of $\mathrm{Mg}$ dopant, but during the growth of high temperature p-type layers, $\mathrm{Mg}$ atoms could be easily diffused from surface to QW action region and Lee et al[17] observed that this diffusion could be accelerated along dislocation defects and one of another optical gradual degradations would operate under high operation temperature and voltage. 


\subsubsection{Package level degradation}

Packaging is considered as a low cost method to realize mass production of LEDs and protect LED chips from surrounding damages, including electrostatic discharge (ESD), moisture, high temperature, chemical corrosion, and mechanical shock. As shown in previous research results, the most common failure mechanisms are; (1) Interface delamination which could result in circuit open or heat dispersion problems; (2) Epoxy or Silicone lens darken which worsens the chromatic properties of white LEDs;

\section{(1) Interface delamination failures}

Interface delamination, as one of the common failures encountered during electronic packaging, could threaten the packages' electrical and thermal management. $\mathrm{Hu}$ et al[18] reported the mechanisms of delamination in LEDs packages and compared the two failure driving forces ( thermalmechanical-stress and hygro-mechanical-stress) to accelerate the development of delaminations. By physical analysis, the thermal-mechanical induced stress between layers comes from mismatching Coefficient of Thermal Expansion (CTEs) and specific heat of different materials (Equation 3). And the different capacities of hygroscopic swelling (CME, Coefficient of Moisture Expansion) contribute to generate the hygro-mechanical-stress (Equation 4). So overall, common delaminations either driven by thermal-mechanical-stress or hygro-mechanical-stress will produce voids within interface layers, higher the thermal resistances and finally block the thermal pass, especially for the chip-submount layer and submount-heat sink layer, the major heat dissipating route in this package.

$$
\begin{gathered}
\sigma=E \alpha\left(T-T_{r e f}\right) \\
\sigma=E \beta\left(C-C_{r e f}\right)
\end{gathered}
$$

where $E$ is elastic modulus, $\alpha, T$ and $T_{\text {ref }}$ are CTE, temperature and reference temperature, respectively, $\beta, C$ and $C_{r e f}$ are $\mathrm{CME}$, moisture concentration and relative moisture concentration. $T_{i}$ and $T_{0}$ is the highest junction temperature and ambient temperature, respectively, and $Q$ is the input thermal power.

\section{(2) Epoxy or Silicone lens failure}

The chromatic properties of LED BLUs are determined both by the stability of luminous output produced by chips and by the capability of light penetration which is controlled by the quality of the lens. The Epoxy (Luxeon ${ }^{\mathrm{TM}}$ side-emitter devices) or silicone (MULTILED LRTB G6SG) lenses are applied to the LEDs packages to increase the amount of light emitted to the front[19]. Because being exposed to the air, lenses suffer thermal and moisture cycle, aging during operation time and some crack or flocculent were observed in the aging test, which lower the light output from the chip. In conclusion, the above mechanism is associated with material's chemical degradation within the lifetime, so in order to increase the lifetime of lenses and silicone glue coatings in the LEDs packages, choosing the thermal, mechanical, and chemical stable materials will be the critical step during packaging design.

\subsubsection{Strip system level degradation}

To satisfy the luminance level for LCD screens (typically $200 \mathrm{~cd} / \mathrm{m} 2$ for monitor applications and $500 \mathrm{~cd} / \mathrm{m} 2$ for largearea television applications), several LED BLUs are soldered on the surface of MCPCB by SMT technology to construct a LED backlight strip (Fig 7)[1].

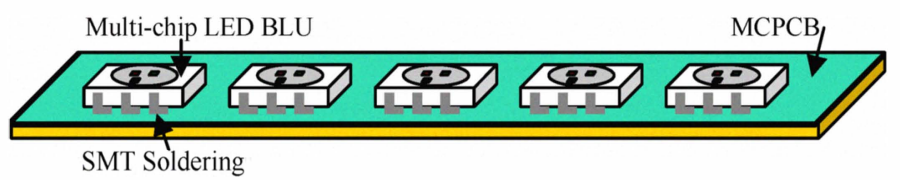

Fig 7 LED backlight strip

Due to the mismatching of the Coefficient of Thermal Expansion (CTE) between solder and lead frame of LED BLUs, temperature cycling could lead to thermal fatigue failures in soldering interconnects[20]. There are two major components to fatigue failures: the initiation of fatigue cracks and the propagation of these cracks under cyclic loading and both of them could cause circuit open and light-off.

\subsection{Failures in driving system section}

LED backlight driving system is a board level electronic assembly which contains active electronic components (i.e. Metal oxide silicon field effect transistors MOSFETs, Bipolar Transistors and Rectifiers et al) and passive components (i.e. capacitors, inductors and resistors et al) and FR-4 PCB[22].

Board level electronic assembly failures can be categorized to: (1) component level degradations; (2) interconnect level failures.

\subsubsection{Component level degradation}

For active components, both MOSFETs and bipolar transistors (BT) are electronic transistors to switching, and are linear LED driver circuits. And the definition of transistor degradation or lifetime is in terms of the percentage shift of threshold voltage, change in transconductance, or variation in driver or saturation current[23].

There are many different effects that take part in MOSFETs' performance degradation in the aging time, among which are hot carrier inject, and charge trapping are two critical effects that contribute to this degradation mechanism[24].

(1) Hot carrier is an electron (or hole) in an active layer that has a higher temperature than the lattice structure and extra carriers generated by hot carrier traveling in the channel region cause impact ionization and avalanche multiplication (Fig 8). In details, the typical effect of hot carrier is to reduce the on-state current in the n-MOSFET and increase the offstate current in the p-MOSFET.

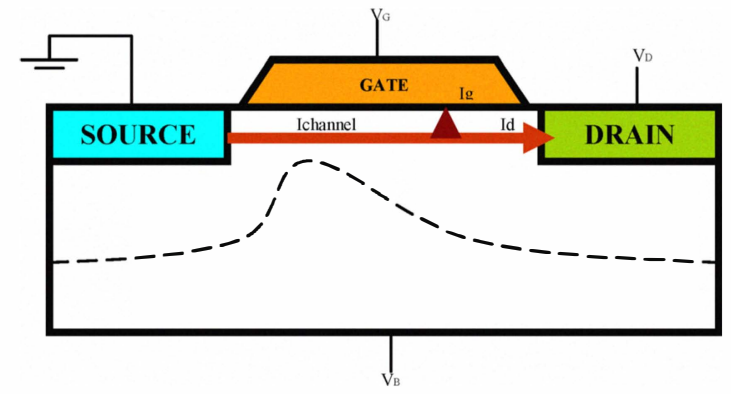

Fig 8 Hot carrier injection effects in MOSFETs

(2) Charge trapping means that the charged carriers are captured at the interface generated by collision and undergoes recombination which leads to the dropping of local charge.

As for passive components (resistors, capacitors, inductors and so on), they are more reliable than active ones within 
electric protection. Therefore, we can ignore their degradations in the whole electronic systems.

\subsubsection{PCB assembly level degradation}

As shown in Fig 4, there are several components mounted on the surface of FR-4 PCB. Deferent package types of components determine the different interconnecting modes. For example, gull wing-type dual in-line (DIP) package for transistors, leadless solder adhesive for resistors, and capacitors and through-hole soldering for capacitors. But the common failure mechanisms among them can be categorized into three types: Chemical based (corrosion, dendritic growth and tin whisker formation), Thermal induced (solder joint fatigue under thermal cycling ) and Mechanical based (solder joint fatigue caused by vibration)25].

(1) Chemical based failures

Due to metal materials for leads or solders, corrosion reaction may be carried out when they are exposed to moisture in the environment. Excessive corrosion may result in strengthless and electric-off failures between components and FR-4 PCB and finally lower the power supply capacity of the driving systems. Dendritic growth, which is a metal migration phenomenon from anode to cathode, can cause the electrical short when complete bridging. And another suspect to result in short circuit is the whisker formation which is commonly seen in tin-plated leads. Previous research showed that residual stresses during plating processes contributed to the formation of whisker and increasing pressure could accelerate its growth at room temperature.

(2) Thermal induced failure

The cyclic thermal loads can cause solder joint fatigue and finally result in interconnect crack, because of different thermal expansion of components and FR-4 PCB. This failure mainly determined by the material properties and geometry of lead.

\section{(3) Mechanical based failure}

External vibrations loading on board can also lead to solder joint fatigue and many models have been developed to calculate vibrational fatigue life. For instant, $\mathrm{Gu}$ et al used PHM method to conduct a reliability assessment for electronic board with components under vibration conditions and obtained a similar life result compared to the experiment[26][27].

\section{CONCLUSIONS AND PROPOSALS}

This paper summarized the failure modes and potential failure mechanisms in LED backlight systems, including LED BLU section and driving system. To complete prognostic and health management to this electronic system, the next step will focus on the following three points:

(1) Rank priority for the identified failure mechanisms based on the field failure data and failure analysis results;

(2) Establish physics-of-failure damage models for the potential failure mechanisms with high priorization.

(3) Select and set loading parameters, profiles and monitored sites for accelerated operations to get failure data and calculate accelerated factor.

(4) Assess and predict the remaining useful lifetime for the whole electronic system

\section{ACKNOWLEDGMENTS}

The work described in this paper was partially supported by a grant from the Research Grants Council of the Hong Kong Special Administrative Region, China (CityU8/CRF/09).

\section{REFERENCE}

[1] Shunsuke Kobayashi, LCD Backlights[M], WILEY, Series in Display Technology, UK, 2009.pp187-205.

[2] Huang-Jen Chiu, LED Backlight Driving System for Large-Scale LCD Panels[J], IEEE TRANSACTIONS ON INDUSTRIAL ELECTRONICS, VOL. 54, NO. 5, OCTOBER 2007, pp2751-2759.

[3] http $/ /$ www.sony.com/

[4] http://www.philipslumileds.com/

[5] http:/www.osram-os.com

[6] M. Day. "LED-driver Considerations," Analog Application Journal, pp. 14-17, 2004

[7] Yu-Kang Lo, Design and Implementation of RGB LED Drivers for LCD Backlight Modules[J], IEEE TRANSACTIONS ON INDUSTRIAL ELECTRONICS, VOL. 56, NO. 12, DECEMBER 2009, pp4862-4871.

[8] M. Ohring, "Reliability and Failure of Electronic Materials and Device"[M], Academic Press, USA, 1998, pp17 20.

[9] M. Pecht. "Integrated Circuit, Hybrid, and Multichip Module Package Design Guidelines: A Focus on Reliability"[M]. Wiley-Interscience Publication. New York. 1993.ppl 39

[10] M. Pecht, J. Gu, "Physics-of-failure-based prognostics for electronic products"[J]. Trans Inst Meas Control 2009:31(3/4): pp309-222.

[11] M. Modarres, "Reliability Engineering and Risk Analysis: A Practical Guide. Second Edition"[M], CRC Press, USA, 2010, pp190-202.

[12] D.H. Stamatis, "Failure Mode and Effect Analysis: FMEA from Theroy to Execution, Second Edition"[M], ASQ Quality Press, USA, 2003, pp275285.

[13] G Meneghesso, M Meneghini, E Zanoni, "Recent results on the degradation of white LEDs for lighting"[L], J. Phys. D: Appl. Phys. 43 (2010) 354007 (11pp)

[14] E. Fred Schubert, "Light-Emitting Diodes"[M], Cambridge University Press, UK, 2003, pp26-46.

[15] A. Uddin, A.C. Wei, T.G. Andersson, "Study of degradation mechanism of blue light emitting diodes"[J], Thin Solid Films 483 (2005), pp378-381

[16] X.A.Cao, P.M. Sandvik, S.F.LeBoeuf, "Defect generation in InGaN/GaN light-emitting diodes under forward and reverse electrical stresses" [J], Microelectronics Reliability 43 (2003) pp1987-1991.

[17] S.N Lee, "Effects of Mg dopant on the degradation of InGaN multiple quantum wells in AlInGaN-based light emitting devices"[J], J Electroceram (2009) 23:pp406-409

[18] J.Z. Hu, "Mechanism and thermal effect of delamination in light-emitting diode packages"[J], Microelectronics Journal 38 (2007) pp157-163.

[19] Y.C. Hsu, Y.K. Lin, M.H. Chen, "Failure Mechanisms Associated With Lens Shape of High-Power LED Modules in Aging Test"'J]. IEEE TRANSACTIONS ON ELECTRON DEVICES,VOL. 55, NO. 2 , FEBRUARY 2008, pp689-693.

[20] John H. Lau, "Solder Joint Reliability: Theory and Applications"[M], Van Nostrand Reinhold, New York, 1991, pp384 405.

[21] http://www.spectrah.com

[22] S. Winder, "Power Supplies for LED Driving[M]", Elsevier/Newness, USA, 2008,pp175-195.

[23] M. Ohring, "Reliability and Failure of Electronic Materials and Devices[M]", Academic Press, USA, 1998, pp330-338.

[24] V. Kaytaz, "A New Simulation Model of Coupled Electro-Thermal Performance for MOSFET Devices”, Istanbul Technical University, 2005 pp47-71

[25] M. Pecht, "Integrated Circuit, Hybrid, and Multichip Module Packaging Design Guidelines: A Focus on Reliability[M]", John Wiley \& Sons, INC, USA, 1994, pp309-315.

[26] J. Gu, "Health Monitoring and Prognostics of Electronics Subject to Vibration Load Conditions[J]", IEEE SENSORS JOURNAL, VOL. 9, NO. 11, NOVEMBER 2009,pp1479-1485.

[27] J. Gu, "Prognostics implementation of electronics under vibration loading[J]", Microelectronics Reliability 47 (2007) pp1849-185 